\title{
Boundary Values of Analytic Functions. II
}

\author{
Florin Constantinescu \\ Department of Theoretical Physics, University of Cluj, Romania
}

Received December 10, 1967

\begin{abstract}
It is known that a complex-valued continuous function $S(x)$ and a Schwartz distribution can both be extended to an analytic function $\hat{S}(z)$ in the complex plane minus the support of $S$. Conditions are given for the existence of limits $\lim _{\varepsilon \rightarrow 0+} \hat{S}(x+i \varepsilon)$, in the ordinary sense, at certain points of the support of $S$, for the case in which $\hat{S}(z)$ is the Cauchy representation. In this way we obtain "local" Plemelj and dispersion relations. Possible generalizations and applications are discussed.
\end{abstract}

\section{\$1. Introduction}

Let $S(x)$ be a complex-valued continuous function on the real line $R$. Given such a function $S(x)$ we can find two functions $\hat{S}_{1}(z), \hat{S}_{2}(z)$ analytic in the upper and lower half-planes respectively $[1,2]$ such that the discontinuity on the real axis is exactly equal to $S(x)$ :

$$
\lim _{\varepsilon \rightarrow 0+}\left[\hat{S}_{1}(x+i \varepsilon)-\hat{S}_{2}(x-i \varepsilon)\right]=S(x) .
$$

It is also known $[1,2]$ that this extends to distributions $S \in \mathscr{D}^{\prime}$ in the sense that there exist analytic functions $\hat{S}_{1}(z), \hat{S}_{2}(z)$ analytic in the upper and lower half-planes respectively such that

$$
\lim _{\varepsilon \rightarrow 0+} \int_{-\infty}^{+\infty}\left[\hat{S}_{1}(x+i \varepsilon)-\hat{S}_{2}(x-i \varepsilon)\right] \cdot \varphi(x) d x=\langle S, \varphi\rangle
$$

for all $p \in \mathscr{D}$. For simplicity, in what follows, we shall write $\hat{S}$ for both $\hat{S}_{1}$ and $\hat{S}_{2}$. If the support of $S(x)$ is not the whole real line, then $\hat{S}(z)$ can be regarded as an analytic function in the entire complex plane minus the support of $S(x)$.

A summary of these results with a bibliography can be found in the introduction of [3]. In the same paper it is shown that in the case of distributions the existence of the limit (1) is possible for certain points of the support of $S$. In this way we can study certain "local" properties of distributions such as a value of a distribution at a point. On the other hand, as has been shown in [4], such "local" properties of distributions are important when discussing unitarity and analyticity of the scattering amplitude. For example, the partial waves $f_{l}(s)$ are distributions. But under certain hypotheses it can be shown that $f_{l}(s)$ is just a Lebesgue measurable function [5], so that there is no difficulty with 
the unitarity relation

and we have

$$
\operatorname{Im} f_{l}(s) \geqq\left|f_{l}(s)\right|^{2},
$$

$$
f_{l}(s)=\lim _{\varepsilon \rightarrow 0+} f_{l}(s+i \varepsilon)
$$

for almost all s. Apparently, there is some interest in "local dispersion relations".

In this paper we intend to study certain "local" properties of distributions. Dispersion relations and relations of the form (1) are both consequences of the Plemelj relations [6].

$$
\begin{aligned}
\lim _{\varepsilon \rightarrow 0+} \hat{S}(x+i \varepsilon) & =\frac{1}{2} S(x)+\frac{1}{2 \pi i} S(x) * P \frac{1}{x} \\
& =-\frac{1}{2} S(x)+\frac{1}{2 \pi i} P \int_{-\infty}^{+\infty} \frac{S(t)}{t-x} d t \\
\lim _{\varepsilon \rightarrow 0+} \hat{S}(x-i \varepsilon) & =-\frac{1}{2} S(x)+\frac{1}{2 \pi i} S(x) * P \frac{1}{x} \\
& =-\frac{1}{2} S(x)+\frac{1}{2 \pi i} P \int_{-\infty}^{+\infty} \frac{S(t)}{t-x} d t,
\end{aligned}
$$

where $\hat{S}(z)$ represents the Cauchy integral of the function $S(x)$. Hence, in $\S 2$ we shall examine the validity of relations of type (3) for distributions. The distribution space $\sqrt{1+t^{2}} \mathscr{D}_{L^{1}}^{\prime}$, introduced in [7], provides the most adequate frame for this problem. In $\S 3$ we will derive "local" dispersion relations for distributions and add some concluding remarks in $\S 4$.

\section{$\$ 2$. Plemelj Relations}

Definition [7]. $\sqrt{1+t^{2}} \mathscr{D}_{L^{1}}^{\prime}$ is the space of those distributions $S$ for which $\frac{S}{\sqrt{1+t^{2}}} \in \mathscr{D}_{L^{1}}^{\prime}[8]$. We say that $S$ converges to zero in $\sqrt{1+t^{2}} \mathscr{D}_{L^{1}}^{\prime}$ when $\frac{S}{\sqrt{1+t^{2}}}$ converges to zero in $\mathscr{D}_{L^{1}}^{\prime}{ }^{1}$.

The distribution spaces $\mathscr{D}, \mathscr{S}, \mathscr{E}^{\prime}, \mathcal{O}_{C}^{\prime}, \mathscr{D}_{L^{p}}^{\prime}$ for $p<\infty$ are contained in $\sqrt{1+t^{2}} \mathscr{D}_{L^{1}}^{\prime}$, being dense in this space. On the other hand, $\sqrt{1+t^{2}} \mathscr{D}_{L^{1}}^{\prime}$ is contained in $\mathscr{S}^{\prime}$. Note that the important space $\mathscr{E}^{\prime}$ of distributions with compact support is contained in $\sqrt{1+t^{2}} \mathscr{D}_{L^{1}}^{\prime}$, as are the spaces $\mathcal{O}_{\alpha}^{\prime}, \alpha \geqq-1[1]$ which are useful in applications ${ }^{2}$. In this paper we use the space $\sqrt{1+t^{2}} \mathscr{D}_{L^{1}}^{\prime}$ because it is the most general space for which

${ }^{1} \sqrt{1+t^{2}}$ may be replaced by $t+i \varepsilon, \varepsilon \neq 0$ ( $\varepsilon$ cannot be chosen to be zero, because of the singularity in $t=0$ ).

${ }^{2}$ The spaces $\mathcal{O}_{\alpha}^{\prime}$ were implicitly introduced also in [9]. 
we can define the convolution

$$
S(t) * P\left(\frac{1}{t}\right) \quad[7] .
$$

Further it is easy to show [8] the existence of the Cauchy representation

$$
\hat{S}(z)=\left\langle\frac{S}{\sqrt{1+t^{2}}}, \quad \sqrt{1+t^{2}} \frac{1}{t-z}\right\rangle
$$

with the usual properties [1]. Now relation (1) follows from (3), so that to write local Plemelj relations for distributions we must at least ensure the existence of the limit (1) at the point $x$ [3]. More precisely, we shall assume the conditions of Theorem 1 hold.

Theorem 1 [3]. If there exists an integer $n \geqq 0$ and a continuous function $F^{\prime}(t)$ such that $F^{(n)}(t)=S(t)$, the derivatives being taken in the sense of distributions, and $i^{3}$

$$
\lim _{t \rightarrow x} n ! \frac{F(t)}{(t-x)^{n}}=C
$$

with $C$ finite, then the limit (1) for the distribution $S$ exists at the point $x$ and is equal to $C$.

But it is known that for Plemelj relations in the case of functions we need a Hölder condition, so that we shall certainly need one in our case, too. We first prove

Theorem 2. Let $S \in \sqrt{1+t^{2}} \mathscr{D}_{L^{1}}^{\prime}$ and assume that there exists an integer $n \geqq 0$ and a continuous function $F(t)$ such that

a) $S(t)=F^{(n)}(t)$, the derivative being taken in the sense of distributions,

b) the limit (5) exists,

c) the function $\Phi_{x}(t)=n ! \frac{F(t)}{(t-x)^{n}}-C$ satifies a Hölder condition with the index $\mu>0$ at $t=x$.

Then the following limits

$$
\begin{aligned}
& \frac{n !}{i \pi} \lim _{\eta \rightarrow 0+}\left(\int_{-\infty}^{x-\eta}+\int_{x+\eta}^{+\infty}\right) \frac{F(t)}{(t-x)^{n+1}} d t, \\
& \lim _{\varepsilon \rightarrow 0+}[\hat{S}(x+i \varepsilon)+\hat{S}(x-i \varepsilon)]
\end{aligned}
$$

exist and are equal.

Proof. Under the hypotheses of the theorem, invoking the representation (4), we can write

$$
\begin{aligned}
\hat{S}(z)=\frac{1}{2 \pi i}\left\langle\frac{F^{(n)}}{\sqrt{1+t^{2}}}, \sqrt{1+t^{2}} \frac{1}{t-z}\right\rangle & =\frac{n !}{2 \pi i}\left\langle\frac{F}{\sqrt{1+t^{2}}}, \sqrt{1+t^{2}} \frac{1}{(t-z)^{n+1}}\right\rangle \\
& =\frac{n !}{2 \pi i} \int_{-\infty}^{+\infty} F(t) \frac{d t}{(t-z)^{n+1}}
\end{aligned}
$$

\footnotetext{
${ }^{3}$ The Theorems 1 and 2 may also be formulated in a different way [3].
} 
Let us form the sum

$$
\begin{aligned}
& \hat{S}(x+i \varepsilon)+\hat{S}(x-i \varepsilon)= \frac{1}{2 \pi i}\left\langle\frac{S}{V 1+t^{2}}, \sqrt{ } 1+t^{2} \frac{1}{t-x-i \varepsilon}\right\rangle \\
&+\frac{1}{2 \pi i}\left\langle\frac{S}{V 1+t^{2}}, \sqrt{ } 1+t^{2} \frac{1}{t-x+i \varepsilon}\right\rangle \\
&= \frac{n !}{2 \pi i} \int_{-\infty}^{+\infty} F(t)\left[\frac{1}{(t-x-i \varepsilon)^{n+1}}+\frac{1}{(t-x+i \varepsilon)^{n+1}}\right] d t \\
&= \frac{n !}{2 \pi i}\left(\int_{-\infty}^{x-\eta}+\int_{x+\eta}^{+\infty}\right) F(t)\left[\frac{1}{(t-x-i \varepsilon)^{n+1}}+\frac{1}{(t-x+i \varepsilon)^{n+1}}\right] d t \\
&+\frac{n !}{2 \pi i} \int_{x-\eta}^{x+\eta} F(t)\left[\frac{1}{(t-x-i \varepsilon)^{n+1}}+\frac{1}{(t-x+i \varepsilon)^{n+1}}\right] d t .
\end{aligned}
$$

Observing that

$$
\begin{aligned}
& \int_{x-\eta}^{x+\eta}(t-x)^{n}\left[\frac{1}{(t-x-i \varepsilon)^{n+1}}+\frac{1}{(t-x+i \varepsilon)^{n+1}}\right] d t \\
= & \int_{-\eta}^{+\eta} t^{n}\left[\frac{1}{(t-i \varepsilon)^{n+1}}+\frac{1}{(t+i \varepsilon)^{n+1}}\right] d t=0
\end{aligned}
$$

we can write the relation (7) in the form

$$
\begin{aligned}
& \hat{S}(x+i \varepsilon)+\hat{S}(x-i \varepsilon) \\
& =\frac{1}{2 \pi i}\left(\int_{-\infty}^{x-\eta}+\int_{x+\eta}^{+\infty}\right)(t-x)^{n} \Phi_{x}(t)\left[\frac{1}{(t-x-i \varepsilon)^{n+1}}+\frac{1}{(t-x+i \varepsilon)^{n+1}}\right] d t \\
& \quad+\frac{1}{2 \pi i} \int_{x-\eta}^{x+\eta} \Phi_{x}(t)(t-x)^{n}\left[\frac{1}{(t-x-i \varepsilon)^{n+1}}+\frac{1}{(t-x+i \varepsilon)^{n+1}}\right] d t .
\end{aligned}
$$

By condition $\mathrm{c}$ ), we can let $\varepsilon \rightarrow 0+$ in the right hand side of relation (9). Then letting $\eta \rightarrow 0+$ the integral over $(x-\eta, x+\eta)$ tends to zero and the other term also has a limit. Hence

$$
\begin{aligned}
& \lim _{\varepsilon \rightarrow 0^{+}}[\hat{S}(x+i \varepsilon)+S(x-i \varepsilon)] \\
= & \lim _{\varepsilon \rightarrow 0^{+}} \frac{n !}{2 \pi i}\left(\int_{-\infty}^{x-\eta}+\int_{x+\eta}^{+\infty}\right) F(t) \frac{1}{(t-x)^{n+1}} d t .
\end{aligned}
$$

We observe that if $n=0$, the right hand side of (10) coincides with the usual principal value

$$
\frac{1}{i \pi} P \int_{-\infty}^{+\infty} \frac{F(t)}{t-x} d t=\frac{1}{i \pi} P \int_{-\infty}^{+\infty} \frac{S(t)}{t-x} d t .
$$

Similarly in our case we could write

$$
\lim _{\varepsilon \rightarrow 0+}[\hat{S}(x+i \varepsilon)+\hat{S}(x-i \varepsilon)]=\frac{1}{i \pi} P\left\langle S, \frac{1}{t-x}\right\rangle,
$$


which is to be understood in the sense of (10).

We now express $P\left\langle S, \frac{1}{t-x}\right\rangle$ in terms of the distribution $S$.

Lemma 1. Let $P \frac{1}{t}$ denote the principal value, then

$$
P \frac{1}{t} * \frac{1}{t-z}=\operatorname{sgn} y \cdot i \pi \frac{1}{t-z}, \quad z=x+i y .
$$

Proof. Let $t-x=u$ and $y>0$. We have

$$
\begin{aligned}
P \frac{1}{t} * \frac{1}{u-i y} & =P \int_{-\infty}^{+\infty} \frac{1}{u-\xi-i y} \frac{d \xi}{\xi} \\
& =\int_{0}^{\infty}\left[\frac{1}{u-\xi-i y}-\frac{1}{u+\xi-i y}\right] \frac{d \xi}{\xi} \\
& =\int_{0}^{\infty} \frac{1}{u-i y}\left[\frac{1}{u+\xi-i y}-\frac{1}{-u+\xi+i y}\right] d \xi \\
& =\frac{1}{u-i y}[\ln (u+\xi-i y)-\ln (-u+\xi+i y)]_{0}^{\infty} \\
& =\frac{1}{u-i y} \cdot i \pi .
\end{aligned}
$$

Similarly for $y<0$ we obtain

$$
P \frac{1}{t} * \frac{1}{u-i y}=\frac{1}{u-i y}(-i \pi) .
$$

Lemma 2. Let $S \in \sqrt{1+t^{2}} \mathscr{D}_{L^{1}}^{\prime}$. Then the expression

$$
\left\langle\frac{S}{\sqrt{1}+t^{2}}, \sqrt{1}+t^{2}\left(P \frac{1}{t} * \varphi\right)\right\rangle=\left\langle\frac{S}{\sqrt{1}+t^{2}}, \psi\right\rangle
$$

exists for $\varphi \in \mathcal{O}_{-2}{ }^{4}$ and defines a continuous linear functional on $\mathbb{O}_{-2}$ i.e. a distribution which we shall write $S * P \frac{1}{t}=P \frac{1}{t} * S=T$. If the convolution exists in the usual sense, it coincides with (13).

Proof. This Lemma is a modification of Proposition 2 of [7] where it is given for $\varphi \in \mathscr{S}$.

We now prove

Theorem 3. Suppose that the distribution $S \in \sqrt{ } \mathbf{1}+t^{2} \mathscr{D}_{L^{1}}^{\prime}$ satisfies the conditions of Theorem 2. Then the distribution $T=S * P \frac{1}{t}$ has a value at the point $x$ and we have

$$
T(x)=-i \pi P\left\langle S, \frac{1}{t-x}\right\rangle .
$$

${ }^{4}$ We remark that although the expression (13) has a meaning for $\varphi(t)=\frac{1}{t+i \varepsilon}$ (this will interest us very much), a direct calculation shows that (13) has no meaning for general $\varphi \in \mathcal{O}_{1}$, because in this case the result of the majorization is a term of order $t^{-1} \ln t$.

24 Commun. math. Phys., Vol. 8 
Proof. By Lemma 2 we have

and by Lemma 1

$$
\begin{gathered}
\left\langle T, \frac{1}{t-x-i \varepsilon}-\frac{1}{t-x+i \varepsilon}\right\rangle \\
=\left\langle S * P \frac{1}{t}, \frac{1}{t-x-i \varepsilon}-\frac{1}{t-x+i \varepsilon}\right\rangle \\
=-\left\langle S, P \frac{1}{t} *\left[\frac{1}{t-x-i \varepsilon}-\frac{1}{t-x+i \varepsilon}\right]\right\rangle
\end{gathered}
$$

$$
\left\langle T, \frac{1}{t-x-i \varepsilon}-\frac{1}{t-x+i \varepsilon}\right\rangle=-i \pi[\hat{S}(x+i \varepsilon)+\hat{S}(x-i \varepsilon)] \text {. }
$$

By Theorem 2, we can let $\varepsilon \rightarrow 0+$ in (16). We show that this limit is equal to the value of the distribution $T$ at the point $x$. Let $\alpha(t)$ be an infinitely differentiable function with the usual properties of a "bump" function used in distribution theory and whose support contains the point $x$. Consequently, the point $x$ is in the compact support of the distribution $U(t)=\alpha(t) T(t)$ and not in the support of the distribution $V(t)$ $=T(t)-U(t)$. One can easily show (as in the proof of Theorem 1, $\S 5.1$ of [1]) that

$$
\lim _{\varepsilon \rightarrow 0+}\left\langle V, \frac{1}{t-x-i \varepsilon}-\frac{1}{t-x+i \varepsilon}\right\rangle=0
$$

and consequently

$$
\begin{aligned}
& \lim _{\varepsilon \rightarrow 0^{+}}\left\langle T, \frac{1}{t-x-i \varepsilon}-\frac{1}{t-x+i \varepsilon}\right\rangle \\
= & \lim _{\varepsilon \rightarrow 0^{+}}\left\langle U, \frac{1}{t-x-i \varepsilon}-\frac{1}{t-x+i \varepsilon}\right\rangle .
\end{aligned}
$$

On the other hand, $U$ has a compact support so that

$$
\begin{gathered}
\lim _{\varepsilon \rightarrow 0+}\left\langle U, \frac{1}{t-x-i \varepsilon}-\frac{1}{t-x+i \varepsilon}\right\rangle \\
=\lim _{\varepsilon \rightarrow 0^{+}}[\hat{U}(x+i \varepsilon)-\hat{U}(x-i \varepsilon)]=U(x)=T(x)
\end{gathered}
$$

where $\hat{U}(z)$ ist the Cauchy representation. Now if $\hat{T}(z)$ is a representation of $T$ (not necessarily the Cauchy representation) we have (Theorem 2 of $[3])$

$$
\begin{gathered}
\lim _{\varepsilon \rightarrow 0+}[\hat{T}(x+i \varepsilon)-\hat{T}(x-i \varepsilon)] \\
=\lim _{\varepsilon \rightarrow 0^{+}}[\hat{U}(x+i \varepsilon)-\hat{U}(x-i \varepsilon)]=T(x) .
\end{gathered}
$$

Taking Theorem 2 into account, we can conclude

$$
\begin{aligned}
& \lim _{\varepsilon \rightarrow 0+}[\hat{S}(x+i \varepsilon)+\hat{S}(x-i \varepsilon)]=\frac{1}{i \pi} P\left\langle S, \frac{1}{t-x}\right\rangle \\
& =-\lim _{\varepsilon \rightarrow 0+}\left[\widehat{S * P \frac{1}{t}}(x+i \varepsilon)-\widehat{S * P \frac{1}{t}}(x-i \varepsilon)\right] \\
& =\lim _{\eta \rightarrow 0+}\left(\int_{-\infty}^{x-\eta}+\int_{x+\eta}^{+\infty}\right) F(t) \frac{1}{(t-x)^{n+1}} d t .
\end{aligned}
$$


Moreover, we showed in [3]

$$
\lim _{\varepsilon \rightarrow 0+}[\hat{S}(x+i \varepsilon)-\hat{S}(x-i \varepsilon)]=S(x) .
$$

Note, however, that (18) was obtained under weaker hypotheses on the local behaviour of $F(t)$ (without the Hölder condition). This question also arises with the ordinary Plemelj relations.

Adding and subtracting (17) and (18) we obtain

$$
\begin{aligned}
& \lim _{\varepsilon \rightarrow 0+} \hat{S}(x+i \varepsilon)=\frac{1}{2} S(x)+\frac{1}{2 \pi i} P\left\langle S, \frac{1}{t-x}\right\rangle \\
& \lim _{\varepsilon \rightarrow 0+} \hat{S}(x-i \varepsilon)=-\frac{1}{2} S(x)+\frac{1}{2 \pi i} P\left\langle S, \frac{1}{t-x}\right\rangle .
\end{aligned}
$$

These are the local Plemelj relations for distributions and reduce to the ordinary case for $n=0$. We draw attention to the fact that relations (19) hold under weaker hypotheses. For example, we have

Theorem 4. Suppose that the distribution $S \in \sqrt{1+t^{2}} \mathscr{D}_{L^{1}}^{\prime}$ satisfies condition a) of Theorem 2 and also the following conditions

$$
\lim _{\varepsilon \rightarrow x+} n ! \frac{F(t)}{(t-x)^{n}} \quad \text { and } \quad \lim _{\varepsilon \rightarrow x-} n ! \frac{F(t)}{(t-x)^{n}}
$$

exist and equal $C_{+}$and $C_{-}$respectively

c) the functions $\Phi_{x}^{+}(t)=n !-\frac{F(t)}{(t-x)^{n}}-C_{+} \quad$ and

$$
\Phi_{x}^{-}(t)=n ! \frac{F(t)}{(t-x)^{n}}-C_{-}
$$

satisfy Hölder conditions with indices $\mu_{+}>0$ and $\mu_{-}>0$ respectively.

Then the conclusion of Theorem 2 hold.

The proof follows the same lines as that of Theorem 4 of [3].

\section{Dispersion Relations}

From (19) we obtain dispersion relations for the case in which $\lim _{\varepsilon \rightarrow 0+} \hat{S}(x-i \varepsilon)=0$ at the point $x$. Then

$$
\text { or } \quad \begin{aligned}
S(x) & =\frac{1}{i \pi} P\left\langle S, \frac{1}{t-x}\right\rangle=T(x) \\
\operatorname{Re} S(x) & =-\frac{1}{\pi} P\left\langle\operatorname{Im} S, \frac{1}{t-x}\right\rangle \\
\operatorname{Im} S(x) & =\frac{1}{\pi} P\left\langle\operatorname{Re} S, \frac{1}{t-x}\right\rangle .
\end{aligned}
$$

Because the space $\sqrt{1+t^{2}} \mathscr{D}_{L^{1}}^{\prime}$ is contained in $\mathscr{S}^{\prime}$ the condition $\lim _{\varepsilon \rightarrow 0+} \hat{S}(x-i \varepsilon)=0$ is assured, for example, by requiring the Fourier transform of the temperate distribution $S$ to vanish on the negative axis [10]. Relation (20) can be considered as "localizing" the dispersion relations of [7]. Certainly if the distribution $S$ does not belong to $\sqrt{1+t^{2}} \mathscr{D}_{L^{1}}^{\prime}$, dispersion relations of type (20) cannot be written and one can resort 
to subtractions. Such questions may be treated in the space $\left(1+t^{2}\right)^{k / 2} \mathscr{D}_{L^{1}}^{\prime}$, $k>1$ and the resulting relations may be considered as "localizations" of subtracted dispersion relations involving distributions [11].

\section{\$ 4. Concluding Remarks}

We observe once again that the relations we have obtained extend the Plemelj and dispersion relations when local Hölder conditions are imposed on the distributions. However, dispersion relations are also valid in the functional form for distributions $[7,10]$, and this suggests us to write down Plemelj relations where the limit for $\varepsilon \rightarrow 0+$ is taken in the sense of distributions. We shall use Lemma 1 to do this.

Just as it is necessary to study the Cauchy integral in the treatment of singular integral equations and in Riemann Hilbert problems, so it is necessary to study the Cauchy representation in treating singular integral equations involving distributions. Problems of that kind have already been treated in [12]. In particular, the Chew-Mandelstam and Shirkov integral equations belong to this category (because the $f_{l}(s)$ are distributions).

On the other hand, the results we have obtained may be extended to the case of an arbitrary boundary curve in the complex plane (possibly containing the point at infinity) as well as to the case of several variables. Some of these questions will be treated on another occasion.

Acknowledgements The author is grateful to Prof. L. Schwartz for drawing his attention to the space $\sqrt{1+t^{2}} \mathscr{D}_{L^{1}}^{\prime}$ and to Mr. H. WIEsLer for interesting discussions on this subject.

\section{References}

1. Bremermanx, H. J.: Distributions, complex variables and Fourier transforms. Reading, Mass.: Addison-Wesley Publishing Co., Inc. 1965.

2. Gütringer, W.: Fortschr. Physik 14, 483 (1966).

3. Constantinescu, F.: Commun. Math. Phys. 7, 225-233 (1968).

4. Martin, A.: CERN Preprint TH. 727 (1966).

5. In [4] as a private communication from V. GLaser; also GLaser, V., and A. Martin - unpublished.

6. Muskmelishvili, N. I.: Singular integral equations. Moscow: Fizmatgiz 1962.

7. Schwartz, L.: Séminaire Schwartz-Levy. 1956-57, No. 3, Faculté des Sciences de Paris; also Anais da Acad. Brasileira de Ciên 34, 13 (1962).

8. - Théorie des distributions, II. Paris: Hermann 1959.

9. Silvi, J. S.: Proc. Intern. Summ. Inst. Lisbon, 327 (1964).

10. Scirwartz, L.: Medd. Lunds. Univ. Mat. Sem. Suppl. M. Riesz, 196 (1952).

11. TAYlor, J. G.: Ann. Phys. 5, 391 (1958).

12. Cernskir, YU. I.: Uspehi Mat. Nauk. 5 (125), 246 (1965).

Dr. F. Constantinescu

Department of Theoretical Physics

University of Cluj

Cluj, Rumänien 